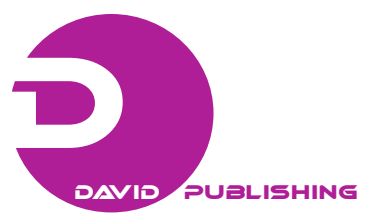

\title{
A Comparative Study of Dynamic Sheath Analytical Models of a Multispecies Plasma Immersion Ion Implantation System in Collisionless Regime
}

\author{
Dushyant Gupta $^{1}$, Jyotika Jogi ${ }^{2}$ and P.J. George ${ }^{3}$ \\ 1. Electronics Department, University College, Kurukshetra University, Kurukshetra 136119, India \\ 2. Department of Physics \& Electronics, ARSD College, University of Delhi, Delhi, India \\ 3. KITM, Kurukshetra University, Kurukshetra, India
}

Received: September 02, 2012 / Accepted: October 08, 2012 / Published: January 25, 2013.

\begin{abstract}
Plasma immersion ion implantation (PIII), a high-dose rate implantation technique, has vast applications in the area of semiconductor electronics, in surface modifications of biomaterials and even in the development of various nano-structures. In this technique, the target is immersed in plasma and the implantation is done by accelerating the ions with a negative pulse voltage, applied to the target. There have been continuous efforts for the development of dynamic sheath models for a PIII system in estimating the impurities to be doped. In this paper, an effort has been made to compare the two models developed for a multispecies collisionless PIII system, one being developed by Qin, et. al. and the other being suggested by the author. To illustrate the comparative study of these two models, various parameters have been computed and compared by considering a mixed plasma of $\mathrm{He}$ and Ar species, when a negative pulse of potential $15 \mathrm{KV}$ and with pulse time of $10 \mu$ s is applied to the sample.
\end{abstract}

Key words: Plasma immersion ion implantation, dynamic sheath, ion plasma frequency, current density, doping concentration.

\section{Introduction}

Plasma immersion ion implantation (PIII) emulates beam line ion implantation technique in a number of areas, viz., high throughput, fast and efficient implantation, independent of wafer topology, by circumventing line-of-sight restrictions and target masking problems of conventional Implantation technique $[1,2]$. In the field of semiconductor electronics, PIII has been successfully implemented in the fabrication of ultra-shallow $\mathrm{p}^{+/ \mathrm{n}}$ junctions, conformal doping of trench sidewall in deep trench-based dynamic RAMs, precise control over gate oxide thickness for memory and logic transistors, formation of Silicon on Insulators (SOI) substrates, poly-silicon thin film transistors (TFTs) for flat-panel

Corresponding author: Dushyant Gupta, associate professor, research fields: process modelling and simulation. E-mail: gupty2kuk@yahoo.co.uk. displays, thin film growth, fabrication of low dielectric constant materials for ULSI multilevel interconnects [3, 4], etc. The researchers are even exploring PIII technique for surface modifications of biomaterials [5-6] and for the development of nanoscale structures, like, carbon nanotubes, $\mathrm{ZnO}$ nanowires, etc. [7-9].

The process is carried out by repetitively applying a high negative voltage pulse to a wafer with respect to the chamber walls, immersed in high-density plasma of appropriate dopant ions. With the negative bias, an initial ion-matrix sheath is formed and the ions start getting implanted into the wafer. After the development of ion-matrix sheath, as the pulse time evolves, ions starts getting implanted into the sample. This phase of sheath expansion plays a crucial role in determining the ion flux to the target. Subsequently, the sheath edge recedes and a nonuniform time varying dynamic sheath expands with time, which contributes 
the major part of the total doping concentration, implanted into the target [10].

For doping of impurities in semiconductors using PIII, one of the major problems is an exact estimation of doping concentration of individual doses in multiple species plasma as the doping source is usually a compound gas such as Phosphine, Borane, etc. and these gases are often diluted in helium and hydrogen for safety purposes and also to control the doping concentration [11]. The dynamic sheath model of multiple-species plasma is, therefore, very important for such applications since undesired impurities too get implanted in addition to the impurities to be doped. To model the PIII processes, a collisionless dynamic sheath model for multispecies plasma was initially suggested by Qin, et al. [12] and subsequently, a generalized analytical model has been suggested by the author [13]. In this paper, a comparative study of the two models has been carried out by computing various parameters for the two models for one pulse duration for a plasma comprising two singly charged ions of $\mathrm{He}$ and Ar.

\section{Collisionless Dynamic Sheath Models}

To determine various parameters like the sheath expansion, total implant ion current, total implant dose and the individual ion doses, the dynamic sheath model developed by Qin, et al. [12], in a two ion species plasma of mass $m_{1}$ and $m_{2}$, the concept of an effective mass, $\mathrm{M}$, which is a function of the plasma composition and ion masses, was considered while in the generalized model being suggested by the author [13], the mass of second ion is considered as $m_{2}=c \cdot m_{1}$, where $c$ is the ratio of the mass $m_{2} / m_{1}$.

The assumptions being considered for both the models are:

- Ion flow is collisionless;

- Electron motion is inertialess during the pulse;

- Planar geometry for target configuration;

- Ideal pulse with no rise time and fall time;

- All ions are singly charged;
- During sheath propagation, the ion density is uniform.

Based on the model of Lieberman, et al. [14], Qin, et al. [12] devised the dynamic sheath expansion for multispecies plasma as under:

$$
\mathrm{s}=\mathrm{s}_{0}\left(\frac{2}{3} \omega_{\mathrm{pi}} \mathrm{t}+1\right)^{1 / 3}
$$

and the ion current density as:-

$$
\mathrm{j}=\frac{2}{9} \frac{\mathrm{en}_{\mathrm{i}} \mathrm{u}_{0}}{\left(\frac{2}{3} \omega_{\mathrm{pi}} \mathrm{t}+1\right)^{2 / 3}}
$$

where $\omega_{\mathrm{pi}}=u_{0} / s_{0}$ is the ion plasma frequency, $\mathrm{s}_{0}=\sqrt{2 \varepsilon_{0} \mathrm{~V}_{0} / \mathrm{en}_{\mathrm{i}}}$ is the initial ion-matrix sheath thickness, $\mathrm{u}_{0}=\sqrt{2 \mathrm{eV}_{0} / \mathrm{M}}$ is the characteristic ion velocity, $n_{\mathrm{i}}$ is the sum of the ion densities of all the species in the plasma and $M$ is the effective mass [12].

The general expression for dynamic sheath expansion being suggested by the author [13] has been derived as:

$$
\mathrm{s}=\mathrm{s}_{0}\left\{\frac{2}{3} \omega_{\mathrm{pi}} \mathrm{tK}+1\right\}^{1 / 3}
$$

and the general expression for ion current density during dynamic sheath propagation for multispecies plasma was suggested as:

$$
\mathrm{j}=\frac{2}{9} \mathrm{~K} \frac{\mathrm{en}_{\mathrm{i}} \mathrm{u}_{0}}{\left(\frac{2}{3} \omega_{\mathrm{pi}} \mathrm{K}+1\right)^{2 / 3}}
$$

Similarly, the general expression for computing individual current density for different species in multispecies plasma was suggested as:

$$
\mathrm{j}_{\mathrm{k}}=\frac{2}{9} \frac{\mathrm{eu}_{0}}{\left(\frac{2}{3} \omega_{\mathrm{pi}} \mathrm{t} \mathrm{K}+1\right)^{2 / 3}} \frac{\mathrm{n}_{\mathrm{k}}}{\sqrt{\mathrm{c}_{\mathrm{k}-1}}}
$$

where

$$
\mathrm{K}=\frac{1}{\mathrm{n}_{\mathrm{i}}} \sum_{\mathrm{k}=1}^{\mathrm{n}} \frac{\mathrm{n}_{\mathrm{k}}}{\sqrt{\mathrm{c}_{\mathrm{k}-1}}} \text { and } \mathrm{c}_{\mathrm{k}-1}=\frac{\mathrm{m}_{\mathrm{k}}}{\mathrm{m}_{1}}
$$

Therefore, in this model, the behavior and the values of dynamic sheath expansion and ion current densities can be estimated by changing the appropriate value of $K$. In case of single ion plasma, the value of $K$ is unity.

The doping concentration of all the ions or of individual ions in the target during Child-law sheath 

Implantation System in Collisionless Regime

propagation for one pulse duration can be computed as [15]:

$$
\mathrm{d}=\frac{1}{\mathrm{e}} \int_{0}^{\mathrm{t}} \mathrm{j}_{\mathrm{m}, \mathrm{c}}(\mathrm{t}) \mathrm{dt}
$$

\section{Results and Discussion}

To illustrate these models, a PIII system with plasma of ion density $n_{\mathrm{i}}=10^{16}$ ions $/ \mathrm{m}^{3}$ for both $\mathrm{He}$ and Ar has been considered. Also a PIII system with a mixture of He and Ar with equal ion density of $5 \times 10^{15} \mathrm{ions} / \mathrm{m}^{3}$ each has been considered. A negative pulse of $10 \mu \mathrm{s}$ duration with $15 \mathrm{KV}$ potential has been applied to the substrate.

The plots for dynamic sheath expansion for plasma of individual ions and also for combined plasma of $\mathrm{He}$ and Ar, shown in Fig. 1, are plotted using Eqs. (1) and (3).

It is observed that the ion-matrix sheath thickness $s_{0}$, being independent of the type of species, is $0.012876 \mathrm{~m}$. Pure helium plasma has a larger sheath expansion velocity than the pure argon plasma, as helium ions are lighter. For dual species plasma, the sheath expansion velocity plots, implementing both the models are between the two plots of pure plasma of He and Ar. It is observed that, implementing the model developed by Qin, et al. [Eq. (1)], the plot of sheath thickness variation with time for combined plasma is closer to that of pure Ar, which should not be the case as the ion velocity of $\mathrm{He}$ is greater. The plot drawn using Eq. (3) appears to be more effective and accurate as it is closer to pure He than that of pure Ar ions as the velocity of He is higher due to its lighter mass.

The ion current density as a function of time for one pulse duration is plotted in Fig. 2 using Eqs. (2) and (4), by putting the value of $K$ and $c$ in it.

In this case, the values of $K$ and $c$ are found to be 0.65769 and 10.0542 , respectively. It is observed that the current density shows the similar dependence on ion mass. Also the mixed plasma of two species, plotted using the model derived by Qin, et al. (Eq. 2), the ion current density plot is closer to that of pure $\mathrm{Ar}$ while in case of the plot drawn using the model

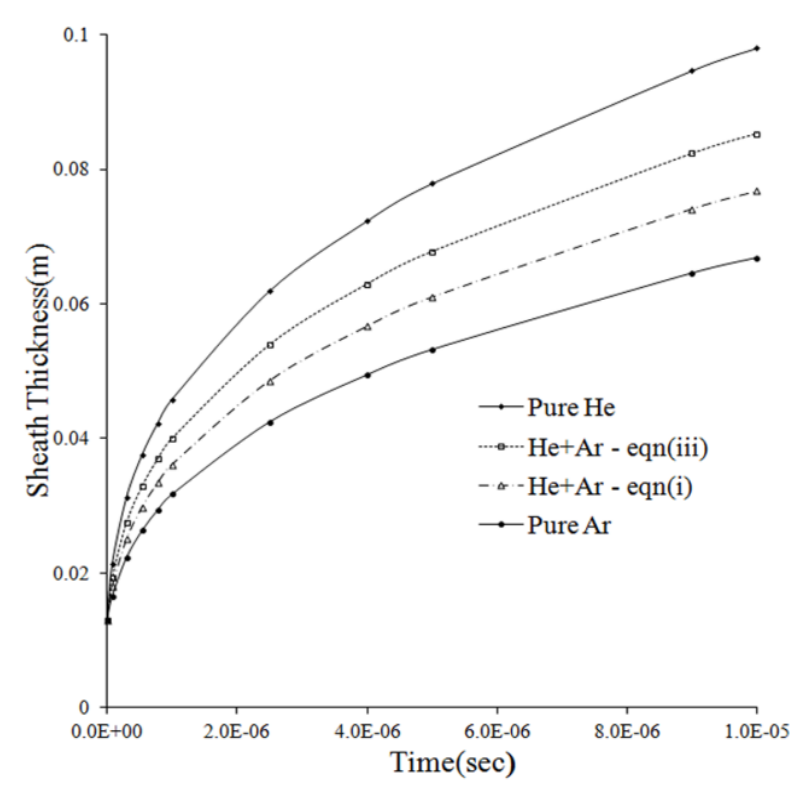

Fig. 1 Comparison of sheath expansion in dual species plasma of $\mathrm{He} \& \mathrm{Ar}$ and in pure Ar \& pure He using two models, during a pulse of $-15 \mathrm{KV}$ potential.

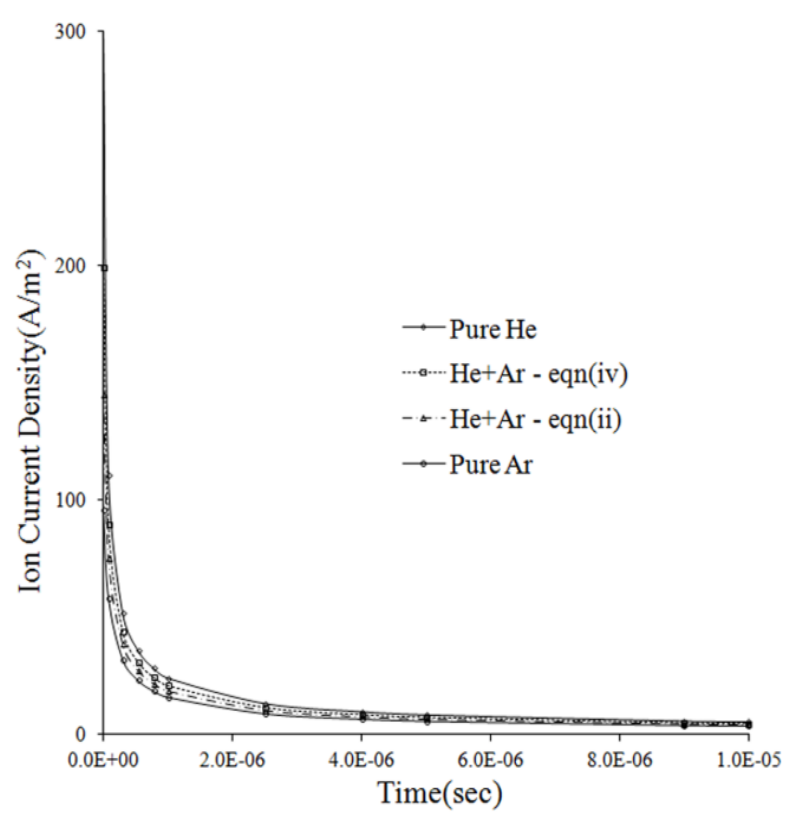

Fig. 2 Comparison of ion current density in dual species plasma of He \& Ar and in pure Ar \& pure He using two models, during a pulse of $-15 \mathrm{KV}$ potential.

suggested by the author (Eq. 4), it is observed that the plot is closer to He ions, which should be the case due to higher ion velocity of He ions with equal ion density.

The two models can also be used to compute the net doping concentration and the respective implant dose components of different ion species during the pulse, despite the fact that, the ions in the sheath have different 


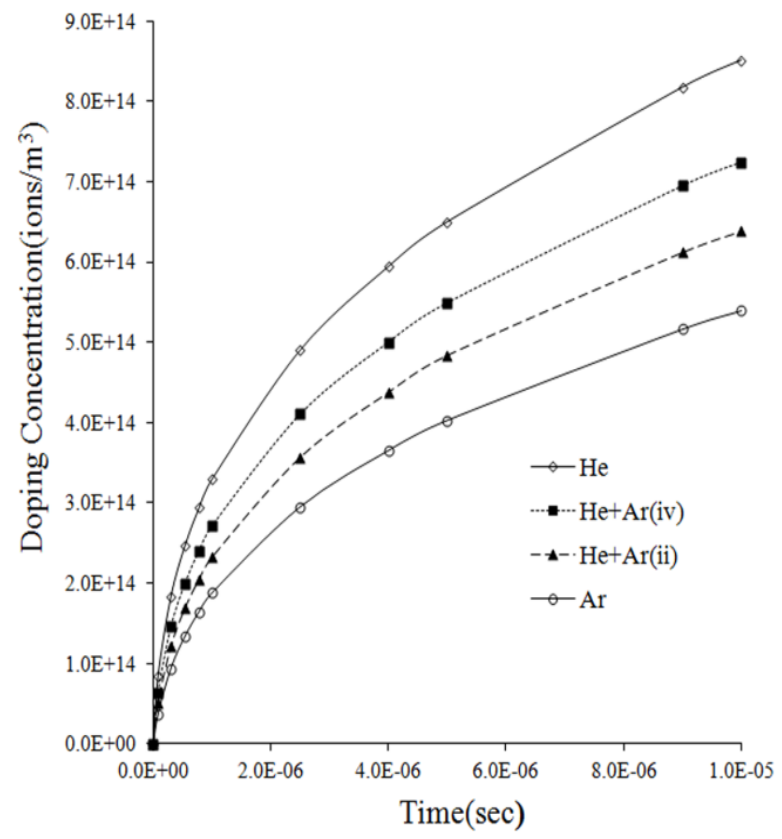

Fig. 3 Comparison of Doping Concentration in dual species plasma of $\mathrm{He} \& \mathrm{Ar}$ and in pure Ar \& pure He using two models, during a pulse of $-15 \mathrm{KV}$ potential.

response times and transit times depending on their masses. In Fig. 3, the net doping concentration for one pulse duration for both the models has been plotted by implementing Eq. (6). It is observed that for a mixed plasma of $\mathrm{He}$ and Ar ions in equal ion density, the plot of net doing concentration, implementing the analytical model of Qin, et al., is closer to that of pure Ar, which should not be the case as $\mathrm{He}$ ions are lighter in mass than Ar ions. For the same case of mixed plasma, when the net doping concentration plot is drawn by implementing the model being developed by the author, as shown in Fig. 3, it is observed that it is closer to pure $\mathrm{He}$ rather than pure Ar curve for He being lighter in mass than Ar and, therefore, He ions will be doped more than $\mathrm{Ar}$ ions in a mixed plasma of both the species having equal ion densities.

While comparing the results of individual doping contributions by $\mathrm{He}$ and $\mathrm{Ar}$ ions, in one pulse duration, in a mixed plasma of both the species with equal ion densities, Qin, et al. has stated through their model that there will be an equal amount of doping contribution by both types of species [12] while the model being developed by the author has proved that the doping concentration contribution of He ions would be $\cong 76 \%$ as compared to $\operatorname{Ar}(\cong 24 \%)$ on account of the lighter mass of He ions [13].

\section{Conclusions}

Comparing the two models for dynamic sheath propagation in a multispecies collisionless PIII system, the model suggested by the author appears to be more accurate and realistic. This model can much accurately estimate and thus monitor the doping concentration of one or more species in comparison with the model suggested by Qin, et al. and can also be helpful in computing the individual contributions of the species in a much effective way, by merely putting an appropriate value of $\mathrm{K}$. Moreover, the model being suggested by the author appears to be more general as it can be implemented in a collisionless PIII system for any number of species for estimating various implanting parameters.

\section{References}

[1] J.R. Conrad, C. Forest, Plasma source ion implantation, in: IEEE International Conference on Plasma Science Saskatoon, Canada, 1986, pp. 28-29.

[2] C. Jones, B.P. Linder, N.W. Cheung, Plasma immersion ion implantation for electronic materials, Jpn. J. Appl. Phys. 35 (1996) 1027-1035.

[3] K. Lee, Plasma immersion ion implantation as an alternative doping tech for ULSI, in: International Workshop on Junction Technology, Japan, 2001, pp. 21-27.

[4] P.K. Chu, Semiconductor applications of plasma immersion ion implantation, Plasma Phy. and Contr. Fusion 45 (2003) 555-570.

[5] N. Huang, P. Yang, Y.X. Leng, J. Wang, H. Sun, J.Y. Chen, et al., Surface modification of biomaterials by plasma immersion ion implantation, Surface and coatings technology 186 (2004) 218-226.

[6] P.K. Chu, Plasma-treated biomaterials, IEEE Trans. on Plasma Science 35 (2007) 181-187.

[7] Z.J. Han, B.K. Tay, M. Shakerzadeh, K. Ostrikov, Superhydrophobic amorphous carbon/carbon nanotube nanocomposites, Appl. Phys. Lett. 94 (2009) 223106-223108.

[8] Y. Yang, X.W. Sun, B.K. Tay, P.H.T. Cao, J.X. Wang, X.H. Zhang, Revealing the surface origin of green band emission from $\mathrm{ZnO}$ nanostructures by plasma immersion 

Implantation System in Collisionless Regime

ion implantation induced quenching, J. Appl. Phys. 103 (2008) 064307-064310.

[9] L. Liao, Z. Zhang, Y. Yang, B. Yan, H.T. Cao, L.L. Chen, et al., Tunable transport properties of n-type $\mathrm{ZnO}$ nanowires by Ti plasma immersion ion implantation, J. Appl. Phys. 104 (2008) 076104-076106.

[10] D. Gupta, B. Prasad, P.J. George, Doping concentration evaluation using plasma propagation models in plasma immersion ion implantation (PIII) system, Solid-State Electronics 48 (2004) 171-174.

[11] S. Qin, C. Chan, Plasma immersion ion implantation doping experiments for microelectronics, J. Vac. Sci. Technol. B 12 (2) (1994) 962-968.
[12] S. Qin, Z. Jin, C. Chan, Dynamic sheath model of collisionless multispecies plasma immersion ion implantation, J. Appl. Phys. 78 (1995) 55-60.

[13] D. Gupta, B. Prasad, J. Jogi, P.J. George, A generalized analytical model of a multispecies plasma immersion ion implantation process in a collisionless system, J. Mater. Sci. Engg. B 1(3) (2011) 372-377.

[14] M.A. Lieberman, Model of plasma immersion ion implantation, J. Appl. Phys. 66 (7) (1989) 2926-2929.

[15] P.K. Chu, S. Qin, C. Chan, N.W. Cheung, L.A. Larson, Plasma immersion ion implantation-a fledging technique for semiconductor processing, Mater. Sci. Eng. R. 17 (1996) 207-280. 